\title{
Tocilizumab effects in COVID-19 pneumonia: role of CT texture analysis in quantitative assessment of response to therapy
}

\author{
Giorgio Maria Masci ${ }^{1}$. Franco lafrate ${ }^{1}$ (D) Fabio Ciccarelli ${ }^{1}$. Giacomo Pambianchi ${ }^{1}$. Valeria Panebianco ${ }^{1}$. \\ Patrizia Pasculli ${ }^{2}$. Maria Rosa Ciardi ${ }^{2}$. Claudio Maria Mastroianni ${ }^{2} \cdot$ Paolo Ricci ${ }^{1}$. Carlo Catalano ${ }^{1} \cdot$ Marco Francone $^{1}$
}

Received: 13 October 2020 / Accepted: 5 May 2021 / Published online: 5 June 2021

(c) Italian Society of Medical Radiology 2021

\begin{abstract}
Purpose To evaluate CT and laboratory changes in COVID-19 patients treated with tocilizumab, compared to a control group, throughout a combined semiquantitative and texture analysis of images.

Materials and methods From March 11 to April 20, 2020, 57 SARS-CoV-2 positive patients were retrospectively compared: group $\mathrm{T}(n=30)$ receiving tocilizumab and group non-T $(n=27)$ undergoing only antivirals/antimalarials. Chest-CT and laboratory findings were analyzed before and after treatment. CT evaluation included both semiquantitative scoring and texture analysis of all parenchymal lesions. Survival and recovery analyses were also provided with Kaplan-Meier method. Results In group T, no significant differences were found for CT score after treatment, while several texture features significantly changed, including mean attenuation $(p<0.0001)$, skewness $(p<0.0001)$, entropy $(p=0.0146)$ and higher-order parameters, suggesting considerable fading of parenchymal lesions. $\mathrm{PaO}_{2} / \mathrm{FiO}_{2}$ mean value significantly increased after treatment, from $240 \pm 93$ to $363 \pm 107(p=0.0003)$, with parallel decrease in inflammatory biomarkers (CRP, D-dimer and LDH). In group non-T, CT scoring, texture and laboratory parameters showed significant worsening at follow-up. Findings were clinically associated with opposite trends between two groups, with reduction of severe cases in group $\mathrm{T}$ (from 21/30 to 5/30; $p<0.0001$ ) as compared to a significant worsening in group non-T (severe cases increasing from $6 / 27$ to $14 / 27 ; p=0.0473$ ). Probability of discharge was significantly higher in group T $(p<0.0001)$, as well as survival rate, although not statistically significant.

Conclusions Our results suggest the potential role of CT texture analysis for assessing response to treatment in COVID-19 pneumonia, using Tocilizumab, as compared to semiquantitative evaluation, providing insight into the intrinsic parenchymal changes.
\end{abstract}

Keywords COVID-19 P Pneumonia · Tocilizumab · Treatment · Computed tomography

\section{Abbreviations}

COVID-19 Coronavirus Disease 2019

SARS-CoV-2 Severe Acute Respiratory Syndrome Coronavirus 2

ARDS Acute Respiratory Distress Syndrome

IL-6 Interleukin-6

$\begin{array}{ll}\text { RT-PCR } & \begin{array}{l}\text { Reverse transcriptase protein chain } \\ \text { reaction }\end{array} \\ \text { CRP } & \text { C-reactive protein } \\ \text { LDH } & \text { Lactate dehydrogenase } \\ \text { SIMIT } & \begin{array}{l}\text { Società Italiana di Malattie Infettive e } \\ \text { Tropicali (Italian Society of Infectious and } \\ \\ \end{array} \quad \text { Tropical Diseases) } \\ \text { Ground-glass opacity }\end{array}$

\section{Introduction}

The worldwide impact of Coronavirus Disease 2019 (COVID-19) outbreak has led to an extensive effort from the scientific community to explore all the therapeutic and prophylactic strategies to stem the spread of the pandemic. 
While supportive care remains the mainstay of therapy, the mechanisms involved in the pathogenesis of Severe Acute Respiratory Syndrome Coronavirus 2 (SARS-CoV2)-induced pneumonia have not been fully understood. On the basis of similar viruses, such as Middle East Respiratory Syndrome Coronavirus (MERS-CoV) and Severe Acute Respiratory Syndrome Coronavirus 1 (SARS-CoV-1), recent studies have hypothesized a primary role of specific immune factors and cytokines in development of acute respiratory distress syndrome (ARDS), which has been found to be the most frequent cause of death in COVID-19 patients [1-3]. More specifically, coronaviruses entry the target cells, mainly pneumocytes, throughout a bond between the viral protein "spike" and the angiotensin-converting enzyme 2 (ACE2) $[4,5]$. After the cell entry and viral replication, a process mediated by antigen-presenting cells (APCs) and major histocompatibility complex (MHC) induces both humoral and cellular immune response, which leads to virus-specific B and T lymphocytes' activation and release of multiple pro-inflammatory cytokines, such as interferon (IFN)- $\alpha$, IFN- $\gamma$, interleukin (IL)-1 $\beta$, IL-6, IL-12, tumor necrosis factor (TNF) $-\alpha$, transforming growth factor (TGF) $-\beta$ and chemokines by immune effector cells [1,3]. This massive immune activation, also known as the "cytokine storm," is believed to be the main responsible of disease progression and outcomes in Severe Acute Respiratory Syndrome (SARS), and it paved the way to new therapeutic options, in addition to the classical antiviral drugs $[2,6,7]$. These unconventional therapies include antimalarial drugs, such as chloroquine and hydroxychloroquine, but also a humanized antiinterleukin-6-receptor (IL-6R) monoclonal antibody currently used as treatment in rheumatoid arthritis, named tocilizumab, that inhibits IL-6 signaling and its off-label use in Italy has been authorized by the Italian Medicines Agency (Agenzia Italiana del Farmaco, AIFA) [8].

Computed tomography (CT) of the chest has a major role as diagnostic tool and also for evaluation of disease progression in COVID-19-related pneumonia, which shows predominately subpleural and basal posterior airspace lesions, with extensive ground-glass opacities and consolidation [9, $10]$.

With regard to the assessment of response to treatment, we hypothesized that multi-feature-based radiomics could allow us to depict subtle CT changes beyond the level of detail accessible to an unaided human eye and may be used to objectively monitor response to therapy in SARS-CoV-2 pneumonia.

Radiomics is an upgrowing discipline that involves the extraction, analysis and interpretation of multiple features from medical images [11]. Texture analysis represents one of the main parts of radiomics, used both in oncological and non-oncological fields, providing an objective and quantitative assessment of tissue heterogeneity by analyzing the distribution and relationship of pixel or voxel gray levels within the image. Texture parameters consist of first-order statistics, which evaluate the gray-level frequency distribution from the pixel intensity histogram in a given area of interest. These include mean attenuation, standard deviation, skewness (asymmetry), kurtosis (peakedness/flatness of pixel histogram), entropy (randomness) and energy (uniformity). Second-order parameters can be based on a co-occurrence matrix (gray-level co-occurrence matrix, GLCM) or can also be calculated on a run-length matrix (gray-level run-length matrix, GLRLM), which analyzes texture in a specific direction. Higher-order statistics, such as contrast, coarseness and busyness, can be calculated using neighborhood gray-tone difference matrices $[12,13]$.

Aim of the study was therefore to evaluate CT and laboratory changes in patients who underwent anti-IL-6R treatment (tocilizumab) and to investigate, throughout texture analysis, the effects of this monoclonal antibody as a potential disease-modifying agent in COVID-19 pneumonia.

\section{Materials and methods}

Ethical approval was obtained for this single-center study and written informed consent was waived due to the retrospective nature of the study.

\section{Study population}

From March 11 to April 20, 2020, all patients with a positive reverse transcription polymerase chain reaction (RT-PCR) for SARS-CoV-2, who underwent chest CT prior and after treatment, were enrolled. Main symptoms and comorbidities were collected. Routine blood tests and arterial blood gas tests were performed for all patients and the following parameters were evaluated: $\mathrm{PaO}_{2} / \mathrm{FiO}_{2}$ ratio, C-reactive protein (CRP), white blood cell count, lymphocyte and monocyte count, D-dimer and lactate dehydrogenase (LDH) levels. Vital parameters, such as respiratory frequency and $\mathrm{O}_{2}$ saturation, were also collected.

Population was divided into two groups, according to different treatment protocols. Particularly, the group of patients who received tocilizumab within their therapy, in addition to antiviral and/or antimalarial drugs, was named "group T"; the group of patients who received only antiviral and/or antimalarial therapy (excluded tocilizumab), was named "group non-T." All parameters and laboratory findings, performed at the same day of both CT scans, were evaluated before and after treatment and were compared for both groups.

Exclusion criteria for this study were: age $<18$ years; no follow-up CT performed after treatment; uncomplete medical data (clinical and laboratory findings) before and 
after treatment. A flowchart of our study population is represented in Table 1.

\section{Treatment protocol}

Therapeutic protocol for all patients was established according to the guidelines provided by the Lombardy section of the Italian Society of Infectious and Tropical Diseases (Società Italiana di Malattie Infettive e Tropicali, SIMIT), as follows: (i) asymptomatic or mildly symptomatic patients were clinically monitored and supportive therapy was provided; (ii) mildly symptomatic patients underwent antiviral therapy with lopinavir/ritonavir or darunavir/cobicistat + chloroquine or hydroxychloroquine; (iii) severe symptomatic patients with ARDS underwent antiviral therapy with remdesivir or lopinavir/ ritonavir or darunavir/cobicistat + chloroquine or hydroxychloroquine [14].

In addition to the aforementioned therapies, tocilizumab was administered according to the following inclusion criteria provided by the SIMIT. Dosage indications for tocilizumab administration were provided by SIMIT according to the Chinese multicenter, randomized controlled trial (ChiCTR2000029765) experienced in Anuhi Hospital [14].

\section{Clinical staging}

Patients were classified in mild, severe or critical disease stage, according to the following criteria provided by the Chinese Center of Disease Control (CDC): (i) mild disease including non-pneumonia or mild pneumonia; (ii) severe disease including dyspnea, respiratory frequency $\geq 30$ / min, blood oxygen saturation $(\mathrm{SpO} 2) \leq 93 \%, \mathrm{PaO}_{2} / \mathrm{FiO}_{2}$ ratio $<300 \mathrm{mmHg}$ and/or lung infiltrates $>50 \%$ within 24 to $48 \mathrm{~h}$; (iii) critical disease including ARDS or respiratory failure, septic shock and/or multiple organ dysfunction (MOD) or failure (MOF). The same clinical staging was provided after treatment and comparisons were made [15].

\section{CT protocol}

Taking into account the uncertainty of clinical evolution and the pharmacodynamic of tocilizumab, a study protocol including 2 CT exams was established in agreement with the Department of Infectious diseases of our hospital [16].

Patients were scanned at admission, and after a follow-up period of 14 days, during hospital stay, using two Multidetector CT scanners (Somatom Sensation 16 and Somatom Sensation 64; Siemens Healthcare, Erlangen, Germany) and appropriate infection prevention and control measures were arranged in all cases, including prompt sanitation of CT

Table 1 Flowchart of the study population

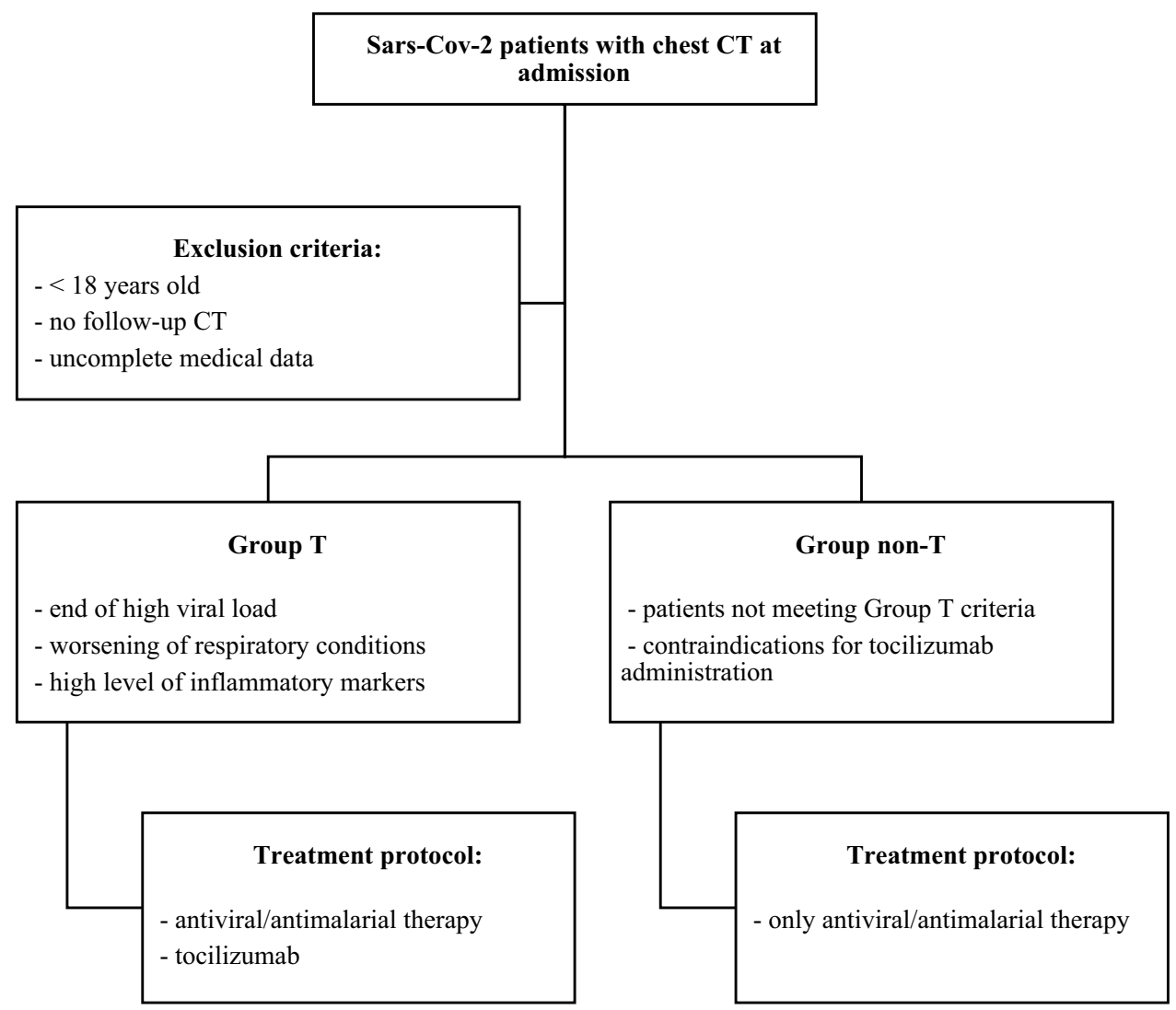


facility and patient's isolation. Acquisitions were performed at end-inspiratory phase with patient in supine position and scanning parameters were as follows: tube voltage: 120 $\mathrm{kVp}$; tube current: 100-250 mAs; pitch: 1.2; collimation: $0.625-0.75 \mathrm{~mm}$. After unenhanced scans were obtained, images were reconstructed using a 1-mm slice thickness in all cases with both soft tissue kernel of B20 and lung kernel of B75. Coronal and sagittal multiplanar reconstructions were also available in all cases.

\section{CT Scoring and texture analysis}

According to the scoring system previously used, a semiquantitative CT severity score was calculated per each of the five lobes, for all patients, considering the extent of lobar involvement ( 0 to 5 ) as follows: 0 , no involvement; 1 , involvement $<5 \%$; $2,6-25 \%$ involvement; $3,26-50 \%$ involvement; 4, 51-75\% involvement; 5, involvement $>75 \%$. A global CT score was also calculated, by summing each of the 5 lobar scores ( 0 to 25$)$ [17, 18].

The following prevalent patterns were also described, based on the standard glossary for thoracic imaging provided by the Fleischner Society: ground-glass opacity (GGO), crazy-paving pattern and pulmonary consolidation [19]. When present, on the basis of previous publications, related imaging findings such as fibrosis (which was considered as the presence of subpleural curvilinear lines, interlobular septa thickening and/or fibrous stripes), pleural effusion and lymphadenopathy (considered when at least one lymph node with short axis $\geq 1 \mathrm{~cm}$ was present) were also described $[9,20,21]$

Lung parenchyma was, additionally, anatomically divided into anterior and posterior zone by drawing a vertical line through the midpoint of the diaphragm in the sagittal reconstruction and distribution of lung abnormalities was also classified as predominately anterior, posterior or both, as well as peripheral (involving mainly the peripheral region comprising one-third of the lung), central (involving mainly the central region comprising two-thirds of the lung), or both peripheral and central [22].

Texture analysis was performed using LIFEx software on lung kernel (B75) reconstructions [23]. For all patients, regions of interest (ROIs) were manually tailored along the margins of all parenchymal lesions for the whole extent of the lesions and volumes of interest (VOIs) were obtained for each lesion. Arterial, venous and bronchial branches were excluded from the ROIs as much as possible. The same VOIs were applied to the same parenchymal area on posttreatment CT examinations in order to evaluate the residual lesions within that VOI and eventual regression of disease in the surrounding area. In case of disease progression, the VOI was first obtained on post-treatment CT and then analyzed on pre-treatment CT. All texture parameters (first-, second- and higher-order statistics) were extracted and analyzed. In addition, volumetric images of affected lungs were obtained using a 3D workstation (Vitrea, Vital Images).

CT scoring and texture analysis were performed by two radiologists both with 15 years of experience and were evaluated prior and after treatment for both groups (Fig. 1).

\section{Statistical analysis}

Data were analyzed using statistical software Prism version 8.3 (GraphPad Software) and SPSS version 26.0 (IBM Corp. SPSS Statistics for Macintosh). Continuous variables were expressed as mean value \pm standard deviation (SD). The Mann-Whitney test was used for single comparisons, while the Kruskal-Wallis test was used for multiple comparisons. The frequencies of demographic and clinical characteristics were expressed as the number (percentage) of occurrences and were compared using the 2-tailed $\chi^{2}$ test or Fisher's exact test, as well as CT patterns and related imaging findings. Follow-up data included patients requiring mechanical ventilation or intubation, evaluation of survival rate and percent of discharged patients in both groups over a 30-day observation period throughout Kaplan-Meier curves. Differences for which $p<0.05$ were considered statistically significant. Intraclass correlation coefficients (ICCs) were used to investigate interobserver variability for texture analysis: ICC values $<0.40$ signified poor agreement; $0.41-0.60$, moderate agreement; $0.61-0.80$, good agreement; $>0.80$, excellent agreement.

\section{Results}

\section{Study population}

From March 11 to April 20, 2020, 57 patients (38 males, 19 females), who had positive RT-PCR for SARS-CoV-2 and underwent $\mathrm{CT}$ before and after any treatment, were enrolled.

Mean age of 62.2 years \pm 11.4 [standard deviation (SD)] (age range 38-80 years). Mean time since symptoms' onset at patients' arrival at our Institution was 7 days \pm 2.5 . Main symptoms were fever $(53 / 57,93 \%)$, coughing $(40 / 57,70.2 \%)$ and dyspnea $(27 / 57,47.4 \%)$. Most frequent comorbidities were arterial hypertension $(22 / 57,38.6 \%)$ and diabetes $(7 / 57,12.3 \%)$. Mean value of respiratory frequency was $21.3 \pm 3.5$ (breaths $/ \mathrm{min}$ ) and mean value of $\mathrm{O}_{2}$ saturation was $94.6 \pm 2.7(\%)$. Laboratory findings showed increased CRP levels (CRP $>0.5 \mathrm{mg} / \mathrm{dL})$ in $55 / 57(96.5 \%)$ patients, increased D-dimer levels (>500 ng/mL) in 48/57 (84.2\%) patients, increased LDH levels (> 225 UI/L) in 53/57 (93\%) patients. Leukopenia was found in 15/57 (26.3\%) patients, decreased lymphocyte count in 41/57 (71.9\%) patients, while decreased monocyte count in 16/57 (28.1\%) patients. 
Fig. 1 Comparison of CT volumetric images and example of texture analysis before and after tocilizumab
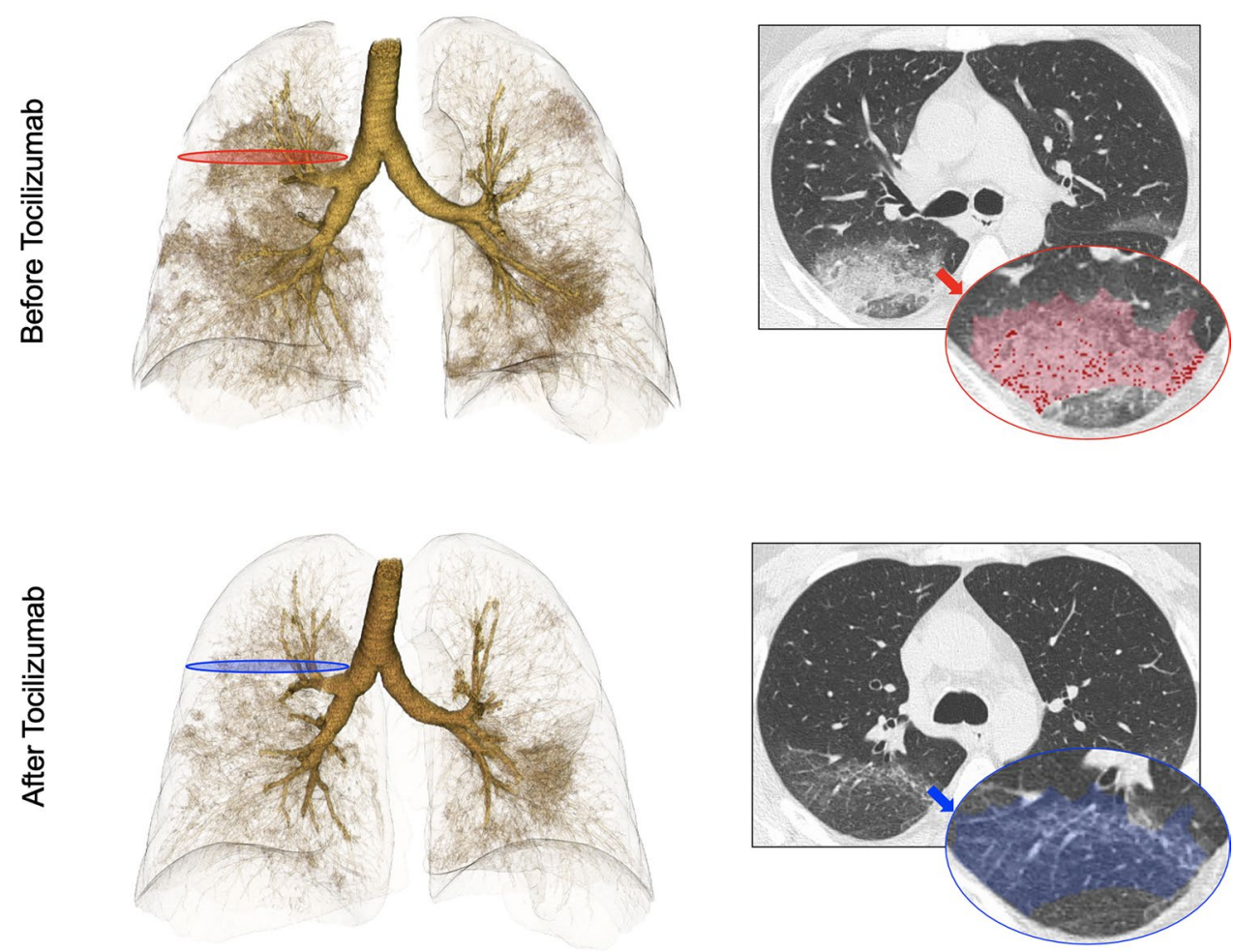

Decreased $\mathrm{PaO}_{2} / \mathrm{FiO}_{2}$ ratio $(<500 \mathrm{mmHg}$ ) was found in 52/57 (91.2\%) patients. Regarding clinical staging, 30/57 (52.6\%) patients were classified as mild, $27 / 57$ (47.4\%) as severe and no patient was classified as critical. Demographic and clinical characteristics of the two groups are summarized in Table 2.

Group $\mathrm{T}$ (who received tocilizumab) consisted of 30 patients (mean age 60.5 years \pm 11.3 ), 9 of which $(30 \%)$ received lopinavir/ritonavir, 5/30 (16.7\%) received darunavir/cobicistat and 27/30 (90\%) also received chloroquine or hydroxychloroquine. Among these patients, 21/30 (70\%) were in severe stage disease and 9/30 (30\%) in mild stage.

Group non-T consisted of 27 patients (mean age 64 years \pm 11.6$), 8$ of which $(29.6 \%)$ received lopinavir/ ritonavir, $7 / 27(25.9 \%)$ received darunavir/cobicistat and all 26/27 (96.3\%) received chloroquine or hydroxychloroquine. The majority of these patients were in mild-stage disease $(21 / 27,77.8 \%)$, while $6 / 27(22.2 \%)$ in severe stage.

\section{CT Changes}

CT scoring and findings were compared before and after 14 days since any treatment was administered (tocilizumab, antivirals and antimalarials for group T; only antivirals and antimalarials for group non-T).

Regarding group T, no significant difference was found for global CT score, with a mean value of $14.5 \pm 4.8$ before treatment, slightly reduced to $12.3 \pm 4$ in the follow-up $(p=0.0904)$. Lower lobes were predominately involved both prior and after treatment. No significant changes were found for lobar score after tocilizumab was administered, except for left lower lobe (LLL) that showed a significantly lower score in post-treatment scan $(p=0.0393)$. No significant difference was found concerning the frequency of prevalent patterns and related imaging findings, except for fibrosis that was significantly more frequent in posttreatment CT $(p=0.0419)$. Same lesion distribution was found before and after treatment.

Regarding group non-T, CT score was significantly higher after treatment without tocilizumab, with a mean value of $8.8 \pm 4.4$ (before) and $12.5 \pm 4.1$ (after) $(p=0.0112)$. Lower lobes were predominately involved in pre-treatment CT both in right lung $(p=0.0301)$ and left lung $(p=0.0088)$, while no significant difference for lobar involvement was found in post-treatment $\mathrm{CT}$ in right $(p=0.0540)$ and left lung $(p=0.3472)$. Left upper lobe (LUL) showed a significantly higher score in posttreatment CT $(p=0.0382)$. Concerning prevalent patterns and related imaging findings, after treatment, GGO was significantly less frequent $(p<0.0001)$, while crazy-paving and fibrosis were significantly more frequent (respectively, $p=0.0214$ and $p<0.0001$ ). After treatment, in addition to peripheral involvement, central distribution was significantly more frequent $(p=0.0008)$ and anterior areas of lungs were significantly more involved compared to pre-treatment CT $(p=0.0473)$. Specific CT findings are reported in Table 3. 
Table 2 Population characteristics

\begin{tabular}{|c|c|c|c|c|}
\hline Characteristic & Total $(n=57)$ & Group T $(n=30)$ & Group non-T $(n=27)$ & $p$ value \\
\hline \multicolumn{5}{|l|}{ Gender } \\
\hline Male & $38 / 57(66.7 \%)$ & $21 / 30(70 \%)$ & $17 / 27(63 \%)$ & 0.5885 \\
\hline Female & $19 / 57(33.3 \%)$ & $9 / 30(30 \%)$ & $10 / 27(37 \%)$ & 0.5885 \\
\hline Age* $^{*}$ & 62.2 years \pm 11.4 & 60.5 years \pm 11.3 & 64 years \pm 11.6 & 0.2767 \\
\hline \multicolumn{5}{|l|}{ Symptoms } \\
\hline Fever $\left(\mathrm{T} \geq 37.5^{\circ} \mathrm{C}\right)$ & $53 / 57(93 \%)$ & $28 / 30(93.3 \%)$ & $25 / 27(92.6 \%)$ & $>0.9999$ \\
\hline Coughing & $40 / 57(70.2 \%)$ & $22 / 30(73.3 \%)$ & $18 / 27(66.7 \%)$ & 0.7725 \\
\hline Dyspnea & $27 / 57(47.4 \%)$ & $19 / 30(63.3 \%)$ & $8 / 27(29.7 \%)$ & 0.0167 \\
\hline \multicolumn{5}{|l|}{ Vital parameters* } \\
\hline Respiratory frequency (breaths/min) & $21.4 \pm 4.6$ & $21.8 \pm 3.6$ & $20.1 \pm 3.2$ & 0.1790 \\
\hline $\mathrm{O}_{2}$ saturation $(\%)$ & $94.6 \pm 2.7$ & $94.1 \pm 2.4$ & $95.6 \pm 3$ & 0.1675 \\
\hline \multicolumn{5}{|l|}{ Comorbidities } \\
\hline Arterial hypertension & $22 / 57(38.6 \%)$ & $9 / 30(30 \%)$ & $13 / 27(48.1 \%)$ & 0.1837 \\
\hline Diabetes & $7 / 57(12.3 \%)$ & $4 / 30(13.3 \%)$ & $3 / 27(11.1 \%)$ & $>0.9999$ \\
\hline \multicolumn{5}{|l|}{ Laboratory findings } \\
\hline $\begin{array}{l}\text { Increased CRP level } \\
(>0.5 \mathrm{mg} / \mathrm{dL})\end{array}$ & $55 / 57(96.5 \%)$ & $29 / 30(96.7 \%)$ & $26 / 27(96.3 \%)$ & $>0.9999$ \\
\hline Increased D-dimer level (> $500 \mathrm{ng} / \mathrm{mL})$ & $48 / 57(84.2 \%)$ & $27 / 30(90 \%)$ & $21 / 27(77.8 \%)$ & 0.2830 \\
\hline $\begin{array}{l}\text { Increased LDH level } \\
(>225 \mathrm{UI} / \mathrm{L})\end{array}$ & $53 / 57(93 \%)$ & $29 / 30(96.7 \%)$ & $24 / 27(88.9 \%)$ & 0.3360 \\
\hline Leukopenia $\left(<4 \times 10^{3} / \mathrm{ML}\right)$ & $15 / 57(26.3 \%)$ & $11 / 30(36.7 \%)$ & $4 / 27(14.8 \%)$ & 0.0768 \\
\hline Decreased lymphocyte count $\left(<1 \times 10^{3} / \mathrm{ML}\right)$ & $41 / 57(71.9 \%)$ & $24 / 30(80 \%)$ & $17 / 27(63 \%)$ & 0.2378 \\
\hline Decreased monocyte count $\left(<0.2 \times 10^{3} / \mathrm{ML}\right)$ & $16 / 57(28.1 \%)$ & $11 / 30(36.7 \%)$ & $5 / 27(18.5 \%)$ & 0.1513 \\
\hline Decreased $\mathrm{PaO}_{2} / \mathrm{FiO}_{2}$ ratio $(<400)$ & $52 / 57(91.2 \%)$ & $27 / 30(90 \%)$ & $25 / 27(92.6 \%)$ & $>0.9999$ \\
\hline \multicolumn{5}{|l|}{ Clinical stage } \\
\hline Mild & $30 / 57(52.6 \%)$ & $9 / 30(30 \%)$ & $21 / 27(77.8 \%)$ & 0.0005 \\
\hline Severe & $27 / 57(47.4 \%)$ & $21 / 30(70 \%)$ & $6 / 27(22.2 \%)$ & 0.0005 \\
\hline Critical & $0 / 57(0 \%)$ & $0 / 30(0 \%)$ & $0 / 27(0 \%)$ & - \\
\hline
\end{tabular}

Except where indicated, data are expressed as number of patients/total patients (percentage). *Data express mean value \pm standard deviation. $T$ : body temperature. $C R P$ : $C$-reactive protein. $L D H$ : lactate dehydrogenase

\section{Texture analysis}

Significant differences were found in group $\mathrm{T}$, when comparing CT features before and after treatment with tocilizumab, among histogram-based (first-order) parameters. Mean attenuation, expressed in Hounsfield units (HU), was significantly lower after tocilizumab with a mean value of $299.33 \pm 200.57 \mathrm{HU}$ (before) versus $-468.58 \pm 227.04 \mathrm{HU}$ (after) $(p<0.0001)$ (Fig. 2). Standard deviation, skewness and entropy were significantly higher after tocilizumab (respectively, $p<0.0001, p<0.0001$ and $p=0.0146$ ). No significant differences were found for kurtosis and energy (Table 5).

In group non-T, mean attenuation was significantly higher after treatment without tocilizumab, with a mean value of $-475.69 \pm 131.97 \mathrm{HU}$ (before) versus $-323.22 \pm 155.38 \mathrm{HU}$ (after) $(p<0.0001)$ (Fig. 2). Standard deviation and entropy were also significantly higher after treatment (respectively, $p=0.0004$ and $p=0.0043$ ), while skewness and energy were significantly lower in post-treatment CT (respectively, $<0.0001$ and $p=0.0405$ ) (Table 4).

Considering also higher-order features, statistically significant differences were found for 28/37 texture parameters in group $\mathrm{T}$ and for $34 / 37$ parameters in group nonT. Specific values of each parameter for both groups are shown in Supplemental Table 1.

\section{Interobserver variability analysis}

Interobserver variability between two radiologists for manual segmentation and texture analysis was calculated using ICC. A good or excellent agreement was found for the majority of texture parameters. The results are shown in Supplemental Table 2. 
Table 3 Comparison of CT features before and after treatment in group $\mathrm{T}$ (treatment with tocilizumab) and in group non-T (treatment without tocilizumab)

\begin{tabular}{llllllr}
\hline Category & $\begin{array}{l}\text { Group T } \\
\text { before }(n=30)\end{array}$ & $\begin{array}{l}\text { Group T } \\
\text { after }(n=30)\end{array}$ & p value & $\begin{array}{l}\text { Group non-T } \\
\text { before }(n=27)\end{array}$ & $\begin{array}{l}\text { Group non-T } \\
\text { after }(n=27)\end{array}$ & $p$ value \\
\hline CT global score* $^{*}$ & $14.5 \pm 4.8$ & $12.3 \pm 4$ & 0.0904 & $8.8 \pm 4.4$ & $12.5 \pm 4.1$ & 0.0112 \\
CT lobar score & & & & & & \\
RUL & $2.6 \pm 1.2$ & $2.3 \pm 1.1$ & 0.3746 & $1.7 \pm 1.3$ & $2.4 \pm 1.2$ & 0.2226 \\
ML & $2.2 \pm 1.2$ & $1.9 \pm 1$ & 0.3675 & $1.1 \pm 0.7$ & $2 \pm 1.1$ & 0.0646 \\
RLL & $3.5 \pm 0.9$ & $2.9 \pm 1$ & 0.0611 & $2.4 \pm 1$ & $3.1 \pm 1.1$ & 0.1293 \\
LUL & $2.6 \pm 1.1$ & $2.2 \pm 1.1$ & 0.2369 & $1.3 \pm 1.3$ & $2.4 \pm 1$ & 0.0382 \\
LLL & $3.7 \pm 1$ & $3.1 \pm 0.9$ & 0.0393 & $2.3 \pm 1.2$ & $2.9 \pm 1.1$ & 0.3317 \\
Prevalent pattern & & & & & & $<0.0001$ \\
GGO & $13 / 30(43.3 \%)$ & $7 / 30(23.3 \%)$ & 0.1702 & $20 / 27(74.1 \%)$ & $5 / 27(18.5 \%)$ & $<14$ \\
Crazy-paving & $9 / 30(30 \%)$ & $12 / 30(40 \%)$ & 0.5889 & $5 / 27(18.5 \%)$ & $14 / 27(51.9 \%)$ & 0.0214 \\
Consolidation & $8 / 30(26.7 \%)$ & $11 / 30(36.7 \%)$ & 0.5796 & $2 / 27(7.4 \%)$ & $8 / 27(29.6 \%)$ & 0.0764 \\
Related features & & & & & & \\
Fibrosis & $21 / 30(70 \%)$ & $28 / 30(93.3 \%)$ & 0.0419 & $9 / 27(33.3 \%)$ & $25 / 27(92.6 \%)$ & $<0.0001$ \\
Pleural effusion & $3 / 30(10 \%)$ & $5 / 30(16.7 \%)$ & 0.7065 & $2 / 27(7.4 \%)$ & $4 / 27(14.8 \%)$ & 0.6687 \\
Lymphadenopathy & $5 / 30(16.7 \%)$ & $5 / 30(16.7 \%)$ & - & $3 / 27(11.1 \%)$ & $4 / 27(14.8 \%)$ & $>0.9999$ \\
Distribution & & & & & & \\
Peripheral & $10 / 30(33.3 \%)$ & $10 / 30(33.3 \%)$ & - & $18 / 27(66.7 \%)$ & $5 / 27(18.5 \%)$ & 0.0008 \\
Peripheral/central & $20 / 30(66.7 \%)$ & $20 / 30(66.7 \%)$ & - & $9 / 27(33.3 \%)$ & $22 / 27(81.5 \%)$ & 0.0008 \\
Lung area & & & & & & \\
Posterior & $16 / 30(53.3 \%)$ & $16 / 30(53.3 \%)$ & - & $14 / 27(51.9 \%)$ & $6 / 27(22.2 \%)$ & 0.0473 \\
Anterior/posterior & $14 / 30(46.7 \%)$ & $14 / 30(46.7 \%)$ & - & $13 / 27(48.1 \%)$ & $21 / 27(77.8 \%)$ & 0.0473 \\
\hline
\end{tabular}

Except where indicated, data are expressed as number of patients/total patients (percentage). *Data express mean value \pm standard deviation. RUL: right upper lobe. ML: middle lobe. RLL: right lower lobe. LUL: left upper lobe. LLL: left lower lobe. GGO: ground-glass opacity

Table 4 Comparison of first-order (histogram-based) texture features before and after treatment in group $\mathrm{T}$ (treatment with tocilizumab) and in group non-T (treatment without tocilizumab)

\begin{tabular}{|c|c|c|c|c|c|c|}
\hline Texture feature & Group T before $(n=30)$ & Group T after $(n=30)$ & $p$ value & $\begin{array}{l}\text { Group non-T before } \\
(n=27)\end{array}$ & Group non-T after $(n=27)$ & $p$ value \\
\hline MA (HU) & $299.33 \pm 200.57$ & $-468.58 \pm 227.04$ & $<0.0001$ & $-475.69 \pm 131.97$ & $-323.22 \pm 155.38$ & $<0.0001$ \\
\hline $\mathrm{SD}(\mathrm{HU})$ & $208.56 \pm 42.96$ & $250.13 \pm 69.42$ & $<0.0001$ & $220.99 \pm 51.53$ & $248.61 \pm 51.66$ & 0.0004 \\
\hline Skewness & $-0.22 \pm 0.69$ & $0.38 \pm 0.84$ & $<0.0001$ & $0.75 \pm 0.72$ & $0.02 \pm 0.86$ & $<0.0001$ \\
\hline Kurtosis & $0.73 \pm 1.40$ & $0.47 \pm 1.33$ & 0.1504 & $0.63 \pm 2.5$ & $0.08 \pm 1.76$ & 0.0723 \\
\hline Entropy & $6.25 \pm 0.32$ & $6.35 \pm 0.39$ & 0.0146 & $6.11 \pm 0.49$ & $6.32 \pm 0.36$ & 0.0043 \\
\hline Energy & $0.016 \pm 0.004$ & $0.015 \pm 0.005$ & 0.1321 & $0.018 \pm 0.008$ & $0.015 \pm 0.005$ & 0.0405 \\
\hline
\end{tabular}

Data are expressed as mean value \pm standard deviation. MA: mean attenuation. SD: standard deviation. HU: Hounsfield unit

\section{Clinical and laboratory changes}

After treatment with tocilizumab, many significant differences were found in group $\mathrm{T}$ among laboratory findings. CRP, D-dimer and LDH levels were significantly lower (respectively, $p<0.0001, p=0.0303$ and $p=0.0014$ ), while lymphocyte and monocyte counts significantly increased up to normal values (respectively, $p<0.0001$ and $p=0.0019$ ), as well as $\mathrm{PaO}_{2} / \mathrm{FiO}_{2}$ ratio $(p=0.0003)$.

Concerning clinical stage, 16 out of 21 patients, who were classified as severe before the beginning of treatment, dropped down to mild stage after 14 days since tocilizumab was administered.

In group non-T, no significant differences were found for any of the evaluated laboratory parameters after treatment. In addition, 8 out of the 21 mild-stage patients were classified as severe after 14 days of treatment without tocilizumab. Clinical and laboratory changes are summarized in Table 5. 


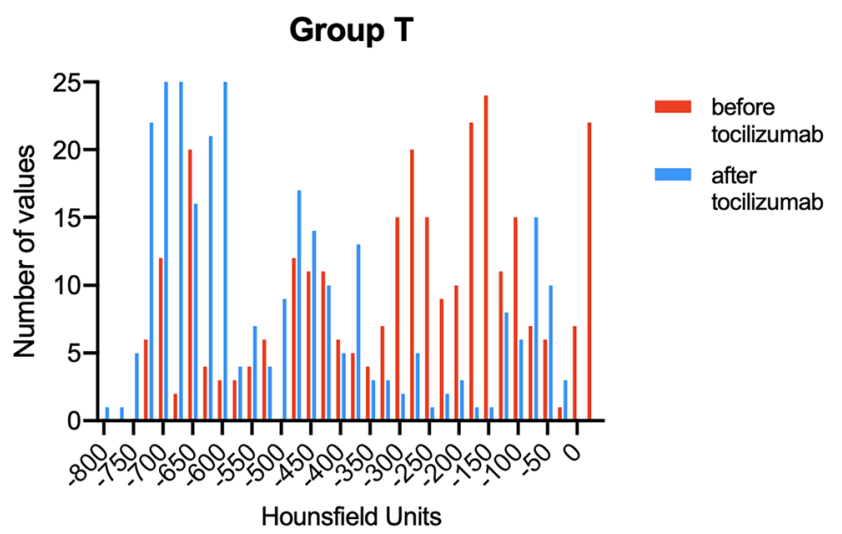

\section{Group non-T}

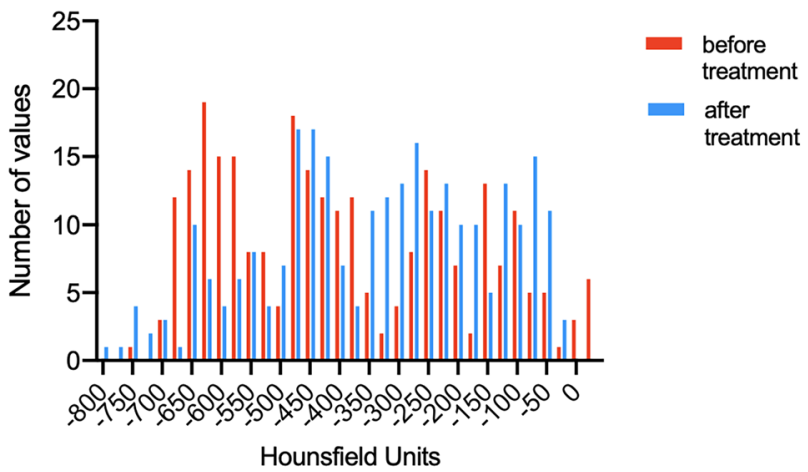

Fig. 2 Histograms of mean attenuation values in before and after treatment in group $\mathrm{T}$ (treatment with tocilizumab) and in group non-T (treatment without tocilizumab)

Table 5 Comparison of laboratory and clinical findings before and after treatment in group $\mathrm{T}$ (treatment with tocilizumab) and in group non-T (treatment without tocilizumab)

\begin{tabular}{|c|c|c|c|c|c|c|}
\hline Characteristic & Group T before $(n=30)$ & Group T after $(n=30)$ & $p$ value & $\begin{array}{l}\text { Group } \\
\text { non-T before } \\
(n=27)\end{array}$ & Group non-T after $(n=27)$ & $p$ value \\
\hline \multicolumn{7}{|c|}{ Laboratory findings (normal range) } \\
\hline $\mathrm{CRP}(0-0.5 \mathrm{mg} / \mathrm{dL})$ & $10 \pm 7.5$ & $0.58 \pm 1.3$ & $<0.0001$ & $6.6 \pm 6.3$ & $7.3 \pm 5.2$ & 0.6842 \\
\hline D-dimer $(0-500 \mathrm{ng} / \mathrm{mL})$ & $2083 \pm 1564$ & $1175 \pm 921$ & 0.0303 & $1725 \pm 1628$ & $1964 \pm 1586$ & 0.5281 \\
\hline LDH (135-225 UI/L) & $359 \pm 156$ & $278 \pm 137$ & 0.0014 & $352 \pm 160$ & $413 \pm 192$ & 0.4813 \\
\hline $\begin{array}{l}\text { Lymphocyte count (1-3.2 } \\
\left.\mathrm{X} 10^{3} / \mathrm{ML}\right)\end{array}$ & $0.84 \pm 0.41$ & $1.42 \pm 0.34$ & $<0.0001$ & $0.9 \pm 0.28$ & $0.96 \pm 0.47$ & 0.9262 \\
\hline $\begin{array}{l}\text { Monocyte count }(0.2-1 \\
\left.\mathrm{X} 10^{3} / \mathrm{ML}\right)\end{array}$ & $0.27 \pm 0.11$ & $0.49 \pm 0.41$ & 0.0019 & $0.27 \pm 0.08$ & $0.28 \pm 0.19$ & 0.8105 \\
\hline $\mathrm{PaO}_{2} / \mathrm{FiO}_{2}$ ratio $(>400)$ & $240 \pm 93$ & $363 \pm 107$ & 0.0003 & $359 \pm 61$ & $302 \pm 73$ & 0.0892 \\
\hline \multicolumn{7}{|l|}{ Clinical stage* } \\
\hline Mild & $9 / 30(30 \%)$ & $25 / 30(83.3 \%)$ & $<0.0001$ & 21/27 (77.8\%) & $13 / 27(48.1 \%)$ & 0.0473 \\
\hline Severe & $21 / 30(70 \%)$ & $5 / 30(16.7 \%)$ & $<0.0001$ & $6 / 27(22.2 \%)$ & $14 / 27(51.9 \%)$ & 0.0473 \\
\hline Critical & $0 / 30(0 \%)$ & $0 / 30(0 \%)$ & - & $0 / 27(0 \%)$ & $0 / 27(0 \%)$ & - \\
\hline
\end{tabular}

Except where indicated, data express mean value \pm standard deviation. *Data are expressed as number of patients/total patients (percentage). CRP: C-reactive protein. $L D H$ : lactate dehydrogenase

\section{Survival and discharge analyses}

Over a period of 30 days during hospital stay, 21 out of 30 patients in group $\mathrm{T}$ required mechanical ventilation, 5 of which underwent endotracheal intubation; in group non-T, 19/27 patients required mechanical ventilation, 6 of which underwent intubation.

Over the same follow-up period, 2 out of 30 patients (6.7\%) died in group $\mathrm{T}$, while 5/27 (18.5\%) died in group non-T (chi-square test, $p=0.2381$ ). Kaplan-Meier curves (Fig. 3) show mean survival of 29.2 days in group $\mathrm{T}$ vs 28.4 days in group non-T (log-rank test, hazard ratio $[\mathrm{HR}]=0.653, p=0.541)$.

\section{Survival}

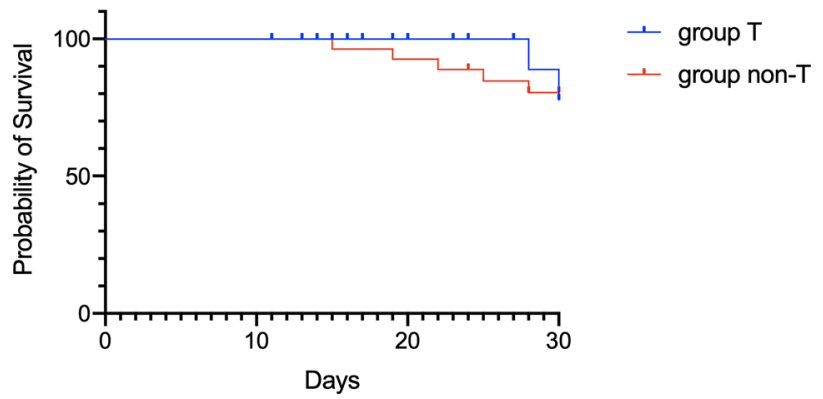

Fig. 3 Kaplan-Meier curves of survival rates in group T (blue) and group non-T (red) 
Moreover, analysis on discharged patients was provided in the two groups. In group T, 22/30 (73.3\%) patients were discharged with a mean time of 19.6 days, while only $7 / 27$ $(25.9 \%)$ patients were discharged in group non-T with a mean time of 26.1 days (chi-square test, $p=0.0005$ ). Probability of discharge was significantly higher in group $\mathrm{T}$ (log-rank test, $\mathrm{HR}=4.261 p=0.0002$ ), according to Kaplan-Meier curve (Fig. 4).

\section{Discussion}

The development and progression of lung injury in COVID19 are mainly driven by a dysregulated immune response to the infection characterized by a sharp increase in the level of a large number of pro-inflammatory cytokines. This process is also known as "cytokine storm" or "cytokine release syndrome" [24].

Use of specific immune-modulating drugs that directly target the cytokines involved in SARS-CoV-2 infection may therefore be helpful to attenuate the inflammatory cascade and improve patients' prognosis.

A key role in the cytokine storm has been attributed to IL-6, which has shown to be significantly increased in severe disease and predictive of fatal outcomes [25]. Use of tocilizumab, a specific monoclonal antibody that selectively blocks IL-6 receptors and inhibits signal transduction, has shown to be promising in the disease management and recommended in severe or critically ill patients [26-28].

Despite its limited clinical validation in COVID-19 pneumonia, tocilizumab is listed in the recommendations for severe COVID-19 management by the National Institute for the Infectious Disease "Lazzaro Spallanzani," which is the national referral for the management of infectious diseases in Italy [29].

Our study aimed to evaluate CT and laboratory changes in COVID-19 patients before and after 14 days of treatment with tocilizumab (group T); results were matched with a

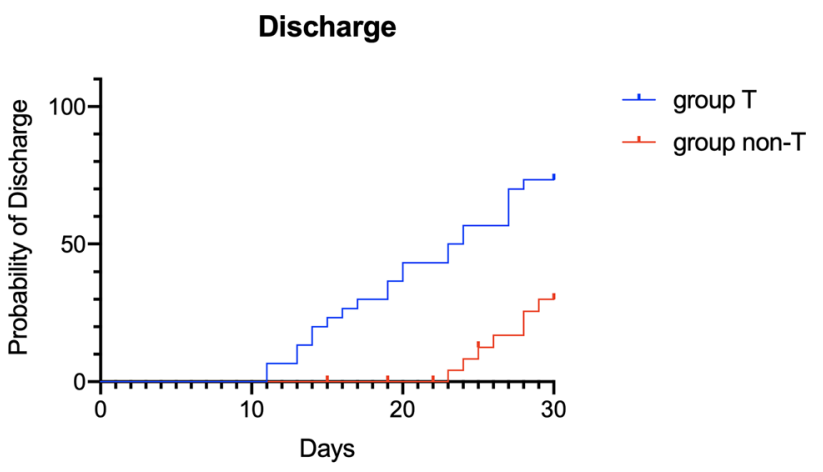

Fig. 4 Kaplan-Meier curves of discharged patients in group T (blue) and group non-T (red) control group which included a cohort of individuals treated with antiviral and antimalarial drugs (group non-T), but not with tocilizumab.

A major selection bias was present among selected groups, which included, respectively, predominately severe stage patients in group $\mathrm{T}$ and mild in group non-T. This is attributable to the inclusion criteria for tocilizumab administration protocol that relies on specific clinical requirements, according to SIMIT guidelines [14]. Except for disease stage, booth groups were homogeneous in terms of demographic and clinical characteristics.

Most important finding of our research is that $\mathrm{PaO}_{2} / \mathrm{FiO}_{2}$ ratio was significantly higher in patients who received tocilizumab compared to those who did not receive tocilizumab, already at 14-day follow-up (Table 5), reflecting a considerable improvement of respiratory function. Similarly, reduced levels of CRP, D-dimer and LDH were observed in group T. Moreover, both lymphocyte and monocyte count also improved after 14 days since tocilizumab was administered.

These findings were clinically associated with a downgrade from severe to mild disease in the majority of group $\mathrm{T}$ patients, while a worsening of disease was noted in group non- $\mathrm{T}$, where severe patients increased from 6 to 14 after therapy.

Chest $\mathrm{CT}$ evaluation and texture analysis of parenchymal lesions added further evidence about the potential efficacy of tocilizumab. One of the challenges in the evaluation of patients undergoing pharmacological treatment concerns quantification of response to therapy.

We assumed that the only semiquantitative evaluation of lung involvement, based on the visual scoring of lobar extent, would have been limited in detecting subtle density changes, as expected in short follow-up assessment. A pure quantitative method derived from the texture analysis on parenchymal abnormalities was therefore applied, in order to evaluate specific pixels and voxels distribution within the lesions. We also analyzed the same radiological and clinical findings in a group of patients who did not receive tocilizumab within their treatment, but exclusively antiviral and antimalarial drugs (group non-T).

In group $\mathrm{T}$, no statistical differences were found for global CT score after treatment, meaning the percentage of the lung involved by the disease was overall not significantly reduced (Table 3). Instead, texture analysis showed significant differences among several first- and higher-order parameters, including mean attenuation, skewness and entropy (Table 4 and Supplemental Table 1), following laboratory changes. More specifically, mean attenuation (expressed as HU) significantly decreased after tocilizumab was administered (Fig. 2) and skewness, which represents the tail of the curve of $\mathrm{HU}$ values in the histogram, significantly increased from negative to positive values (the hump of the curve moved to lower HU values) [12]. The discordance between CT score 
and CT texture analysis could explain how post-treatment lesions become more absorbed, although their extent in lung parenchyma is not significantly reduced. Entropy represents the randomness of pixel distribution within the lesion and the increase in its values after treatment with tocilizumab is probably due to less homogeneity of a faded lesion or fibrotic changes of lung parenchyma compared to a more consolidated pattern visible in pre-treatment CT [13]. Kurtosis is a measure of the curve's peakedness (higher values) or flatness (values close to zero) and its values were close to zero both prior and after tocilizumab, although lower values were found in post-treatment analysis [12]. This could be explained by the spreading of values over different $\mathrm{HU}$ in both curves, mainly in post-treatment $\mathrm{CT}$, confirming less homogeneity of the lesions in this case.

On the other hand, patients who did not receive tocilizumab (group non-T) showed a significant increase in CT score after only antiviral and antimalarial therapy was administered, as well as several texture features. Mean attenuation values were significantly higher after treatment, meaning an increase in lesion density, while skewness significantly decreased from positive values almost to zero. These findings probably underline a progression of disease or a major consolidation process within the lesions. Entropy is the only parameter that seems to be in accordance with the two and that showed a significant increase after treatment, indicating a greater randomness in pixels' disposure, probably related to prevalent fibrotic changes. The significant decrease in energy values also confirms less uniformity of post-treatment lesions in group non-T.

Survival analysis did not show significant differences between the two groups, although occurrence of death was higher in group non-T than in group $\mathrm{T}$ (5/27 vs 2/30) (Fig. 3). On the other hand, when considering patients' discharge from hospital, a significant higher probability of discharge was found among group $\mathrm{T}(22 / 30)$ compared to group non-T (7/27) over a 30-day observational period, with a mean time for discharge, respectively, of 19.6 days vs 26.1 days (Fig. 4). These analyses show that although survival rate is not statistically different among the two groups, use of tocilizumab could shorten hospitalization and recovery time.

On the basis of these preliminary results and according to previous studies, our assumption is that antiviral and antimalarial drugs could not be sufficient as the only treatment for COVID-19 patients [27, 30]. Tocilizumab could have beneficial effects particularly on severe patients, as recently proposed, probably due to massive immune activation characterizing the advanced stages of disease [31]. Moreover, texture analysis, within CT evaluation, could have a promising role in the assessment of disease course and lung changes during follow-up, by objectively quantifying response to therapy. Although patients' management is primarily led by their clinical course and the extraction of radiomic parameters might not be instantaneous, this quantitative analysis can unveil subtle details on the underlining pathological substrate of the lung parenchyma; this can be used not only to establish disease severity but also for the evaluation of the efficacy of treatment protocols.

Our study has several limitations, due to the relatively small size of our sample population in both groups and also to different clinical stages of the two groups before treatment, that did not allow us to validate the effect of tocilizumab in all clinical stages. Moreover, our observation period was limited and therefore potential long-term evaluation, including side effects, could not be considered. IL-6 blood levels were not available: a correlation between these values and response to tocilizumab could have emphasized its role in blocking the immune-related mechanism of COVID-19-induced pneumonia. Finally, considering our survival analysis, other comorbidities or underlying conditions might have influenced mortality and hospital stay; future and larger prospective studies could clarify and overcome these limitations and eventually confirm the usefulness of tocilizumab in this specific viral pneumonia.

\section{Conclusions}

Our preliminary results support the role of texture analysis as a complementary tool for a comprehensive evaluation of CT changes in COVID-19 pneumonia; radiomic features can reveal detailed information on the subtle modifications of lung parenchyma and assess its response to therapy. This may provide to clinicians a complete and fulfilling analysis on the course of disease and may therefore be helpful for patient's management.

Preliminary clinical findings, observed in this selected cluster of patients, suggest a possible role of tocilizumab as a positive disease-modifying agent in severe stages of COVID-19 pneumonia, which shall however be validated in broader analyses.

These considerations regarding use of radiomics in COVID-19 may, obviously, extend beyond this comparative analysis on the effects of tocilizumab.

Supplementary Information The online version contains supplementary material available at https://doi.org/10.1007/s11547-021-01371-7.

Author contributions Giorgio Maria Masci and Franco Iafrate equally contributed.

\section{Declarations}

Conflict of interest The authors of this manuscript declare no relationships with any companies, whose products or services may be related to the subject matter of the article. 
Ethical approval Ethical approval was obtained for this single-center study and written informed consent was waived due to the retrospective nature of the study.

\section{References}

1. Li X, Geng M, Peng Y et al (2020) Molecular immune pathogenesis and diagnosis of COVID-19. J Pharm Anal 10:102-108. https://doi.org/10.1016/j.jpha.2020.03.001

2. Wu C, Chen X, Cai Y et al (2020) Risk factors associated with acute respiratory distress syndrome and death in patients with coronavirus disease 2019 pneumonia in wuhan, china. JAMA Intern Med. https://doi.org/10.1001/jamainternmed.2020.0994

3. Huang C, Wang Y, Li X et al (2020) Clinical features of patients infected with 2019 novel coronavirus in Wuhan, China. Lancet 395:497-506. https://doi.org/10.1016/S0140-6736(20)30183-5

4. Hoffmann M, Kleine-Weber H, Schroeder S et al (2020) SARSCoV-2 cell entry depends on ACE2 and TMPRSS2 and is blocked by a clinically proven protease inhibitor. Cell 181:271-280.e8. https://doi.org/10.1016/j.cell.2020.02.052

5. Zhou P, Yang X-L, Wang X-G et al (2020) A pneumonia outbreak associated with a new coronavirus of probable bat origin. Nature 579:270-273. https://doi.org/10.1038/s41586-020-2012-7

6. Lew TWK, Kwek T-K, Tai D et al (2003) Acute respiratory distress syndrome in critically Ill patients with severe acute respiratory syndrome. JAMA 290:374-380. https://doi.org/10.1001/ jama.290.3.374

7. Zhang Q, Wang Y, Qi C et al (2020) Clinical trial analysis of 2019-nCoV therapy registered in China. J Med Virol. https://doi. org/10.1002/jmv.25733

8. Nishimoto N, Kishimoto T, Yoshizaki K (2000) Anti-interleukin 6 receptor antibody treatment in rheumatic disease. Ann Rheum Dis 59:i21-i27. https://doi.org/10.1136/ard.59.suppl_1.i21

9. Ye Z, Zhang Y, Wang Y et al (2020) Chest CT manifestations of new coronavirus disease 2019 (COVID-19): a pictorial review. Eur Radiol. https://doi.org/10.1007/s00330-020-06801-0

10. Li Y, Xia L (2020) Coronavirus disease 2019 (COVID-19): role of chest CT in diagnosis and management. Am J Roentgenol. https:// doi.org/10.2214/AJR.20.22954

11. Bashir U, Siddique MM, Mclean E et al (2016) Imaging heterogeneity in lung cancer: techniques, applications, and challenges. Am J Roentgenol 207:534-543. https://doi.org/10.2214/AJR.15. 15864

12. Lubner MG, Smith AD, Sandrasegaran K et al (2017) CT Texture analysis: definitions, applications, biologic correlates, and challenges. Radiographics 37:1483-1503. https://doi.org/10.1148/rg. 2017170056

13. Rizzo S, Botta F, Raimondi S et al (2018) Radiomics: the facts and the challenges of image analysis. Eur Radiol Exp 2:36. https://doi. org/10.1186/s41747-018-0068-z

14. Lombardy Section Italian Society Infectious And Tropical Diseases (2020) Vademecum for the treatment of people with COVID-19. Edition 2.0, 13 March 2020. Infez Med 28:143-152

15. Wu Z, McGoogan JM (2020) Characteristics of and important lessons from the coronavirus disease 2019 (COVID-19) outbreak in china: summary of a report of 72314 cases from the chinese center for Disease control and prevention. JAMA 323:1239-1242. https://doi.org/10.1001/jama.2020.2648

16. Dostalek M, Gardner I, Gurbaxani BM et al (2013) Pharmacokinetics, pharmacodynamics and physiologically-based pharmacokinetic modelling of monoclonal antibodies. Clin Pharmacokinet 52:83-124. https://doi.org/10.1007/s40262-012-0027-4

17. Francone M, Iafrate F, Masci GM et al (2020) Chest CT score in COVID-19 patients: correlation with disease severity and short-term prognosis. Eur Radiol. https://doi.org/10.1007/ s00330-020-07033-y

18. Pan F, Ye T, Sun P et al (2020) Time course of lung changes on chest CT during recovery from 2019 novel coronavirus (COVID19) pneumonia. Radiology. https://doi.org/10.1148/radiol.20202 00370

19. Hansell DM, Bankier AA, MacMahon H et al (2008) Fleischner society: glossary of terms for thoracic imaging. Radiology 246:697-722. https://doi.org/10.1148/radiol.2462070712

20. Salehi S, Abedi A, Balakrishnan S, Gholamrezanezhad A (2020) Coronavirus disease 2019 (COVID-19): a systematic review of imaging findings in 919 patients. Am J Roentgenol. https://doi. org/10.2214/AJR.20.23034

21. Glazer GM, Gross BH, Quint LE et al (1985) Normal mediastinal lymph nodes: number and size according to American thoracic society mapping. AJR Am J Roentgenol 144:261-265. https:// doi.org/10.2214/ajr.144.2.261

22. Zhou S, Wang Y, Zhu T, Xia L (2020) CT Features of coronavirus disease 2019 (COVID-19) pneumonia in 62 patients in Wuhan. China Am J Roentgenol. https://doi.org/10.2214/AJR.20.22975

23. Nioche C, Orlhac F, Boughdad S et al (2018) LIFEx: A freeware for radiomic feature calculation in multimodality imaging to accelerate advances in the characterization of tumor heterogeneity. Cancer Res 78:4786-4789. https://doi.org/10.1158/0008-5472. CAN-18-0125

24. Zhang C, Wu Z, Li J-W et al (2020) Cytokine release syndrome in severe COVID-19: interleukin-6 receptor antagonist tocilizumab may be the key to reduce mortality. Int J Antimicrob Agent 55:105954. https://doi.org/10.1016/j.ijantimicag.2020.105954

25. Mehta P, McAuley DF, Brown M et al (2020) COVID-19: consider cytokine storm syndromes and immunosuppression. Lancet 395:1033-1034. https://doi.org/10.1016/S0140-6736(20)30628-0

26. Medicine NHC\& NA of TC (2020) Diagnosis and treatment protocol for novel coronavirus pneumonia (trial version 7). Chin Med J 133:E027-E027. https://doi.org/10.3760/cma.j.issn.0366-6999. 2020.0027

27. Ye Q, Wang B, Mao J (2020) The pathogenesis and treatment of the 'cytokine storm' in COVID-19. J Infect. https://doi.org/10. 1016/j.jinf.2020.03.037

28. Luo P, Liu Y, Qiu L et al (2020) Tocilizumab treatment in COVID-19: a single center experience. J Med Virol 92:814-818. https://doi.org/10.1002/jmv.25801

29. Nicastri E, Petrosillo N, Bartoli TA et al (2020) National institute for the infectious diseases "L. Spallanzani", IRCCS. recommendations for COVID-19 clinical management. Infect Dis Rep 12(1):3-9. https://doi.org/10.4081/idr.2020.8543

30. Zhao M (2020) Cytokine storm and immunomodulatory therapy in COVID-19: role of chloroquine and anti-IL-6 monoclonal antibodies. Int J Antimicrob Agent. https://doi.org/10.1016/j.ijantimicag.2020.105982

31. Fu B, Xu X, Wei H (2020) Why tocilizumab could be an effective treatment for severe COVID-19? J Transl Med 18:164. https://doi. org/10.1186/s12967-020-02339-3

Publisher's Note Springer Nature remains neutral with regard to jurisdictional claims in published maps and institutional affiliations. 